\title{
Influence of SMADI gene in osteoporosis: A bioinformatics approach
}

\author{
Anand Anabarasu \\ Arpita Kundu \\ Bioinformatics Division, School of \\ Biosciences and Technology, VIT \\ University, Tamil Nadu, India.
}

This article was published in the following Dove Press journal:

Open Access Bioinformatics

21 July 2010

Number of times this article has been viewed
Abstract: In the present study we have analyzed the role of SMAD family member 1 protein (SMAD1) gene products in relation to bone morphogenesis and osteoporosis. Out of 1045 single nucleotide polymorphisms (SNP) investigated, we find that one nonsynonymous SNP (nsSNP), rs1804647, to have significant damaging effects as predicted by all the tools used in the analysis. This nsSNP resulted in a change of amino acid from a positive charged residue, Lysine, to a strong negatively charged residue, Glutamate, and hence the change of residue with opposite charges might lead to structural defects and result in altered function. The results presented in this report will be a good starting point for genetic analysis of SMAD1 genes in patients with osteoporosis which might lead to more conclusive evidence of the association of this gene with osteoporosis.

Keywords: osteoporosis, nsSNP, SMAD1, rs1804647

\section{Introduction}

Osteoporosis is a disease associated with progressive deterioration of bone which results in fragility fractures that occur with very little trauma. ${ }^{1,2}$ It is a common disease that affects millions of people worldwide. It is estimated that over 200 million people have osteoporosis. ${ }^{3}$

Genetic factors play a major role in the determination of bone mineral density (BMD) and osteoporosis risk. ${ }^{4}$ Multiple chromosomal loci have been mapped by linkage approaches which potentially carry hundreds of genes involved in the determination of bone mass and quality. Association studies based on candidate gene polymorphisms and subsequent meta-analyses, and the more recent genome-wide association studies, have clearly identified a handful of genes associated with BMD and/or fragility fractures. ${ }^{4}$

Molecular genetic studies have implicated the association of single nucleotide polymorphisms (SNP) with osteoporosis. ${ }^{5}$ It is anticipated that both the diagnosis and the treatment of this disease will be revolutionized by the integration of genomics and informatics. ${ }^{2}$ Also, it is predicted that a genetic algorithm will be developed to identify at-risk patients before they develop osteoporosis, so that preventive measures can be instituted. ${ }^{2}$

The last two decades have seen a tremendous growth of genomic knowledge that is highly relevant to the treatment and management of complex genetic diseases such as osteoporosis. $^{6,7}$ In recent years, bioinformaticians have extracted new and meaningful information from literature. ${ }^{7}$ The bioinformatics resources and tools for systematic analysis of SNPs are well described in a recent review. ${ }^{8}$
Correspondence: Anand Anabarasu Bioinformatics Division, School of Biosciences and Technology, VIT University, Vellore 632014,

Tamil Nadu, India.

Tel +9l 4162202556

Email aanand@vit.ac.in 
The role of SMA- and MAD-related (SMAD) protein in bone morphogenesis is documented by researchers. ${ }^{7,9}$ Even though there is a lot of information on the clinical impact, diagnosis and treatment of osteoporosis, there are not many reports on the systematic analysis of SMAD1 genes in relation to osteoporosis. This report systematically analyzes the SMAD1 gene and its possible implications on osteoporosis using bioinformatics tools. Our results from the present study might be useful for further investigations on the human genetics of osteoporosis.

\section{Materials and methods \\ Data set}

The SNPs associated with SMAD1 gene were obtained from the single nucleotide polymorphism database $(\mathrm{dbSNP})^{10}$ which is a public-domain archive established by NCBI, having broad collection of SNPs of any gene of an organism serving as a public repository for genetic variation.

There are 1045 SNPs associated with SMAD1, these are commonly referred to by their reference sequence IDs (rsID), and they are listed in Table 1.

\section{Predicting damaging nsSNPs using SIFT}

We used the program Sorting Intolerant from Tolerant (SIFT) designed by $\mathrm{Ng}$ and Henikoff to identify the damaging non-synonymous SNP's (nsSNP) in the data set. ${ }^{11,12}$ SIFT predicts whether an amino acid substitution affects protein function and it distinguishes between functionally neutral and deleterious amino acid changes in mutagenesis studies and on human polymorphisms. SIFT also searches for similar protein sequences from different species in the database, obtains the multiple alignments of these sequences, and then calculates from the alignment the tolerance index (from 0 to 1) for all possible substitutions at each position. The higher a tolerance index, the less functional impact a particular amino acid substitution is likely to have. ${ }^{11-13}$

\section{Structural alteration by nsSNPs using PolyPhen}

The structural alteration as a result of nsSNP was determined using the program Polymorphism Phenotyping (PolyPhen). ${ }^{14-16}$ PolyPhen assesses the possible damaging effect of amino acid substitution, based on whether the substitution was

(i) in an active or binding site;

(ii) affecting interactions with ligands present in the experimentally resolved structure; (iii) leading to hydrophobicity or electrostatic charge change in a buried site;

(iv) destroying a S-S bond;

(v) affecting protein's solubility;

(vi) inserting proline $(\mathrm{P})$ in an $\alpha$-helix or

(vii) incompatible with the profile of amino acid substitutions observed at that site in the set of homologous proteins. ${ }^{15}$

\section{Functional analysis and selection for nsSNPs using FASTSNP}

We used the functional analysis and selection tool for single nucleotide polymorphisms (FASTSNP) web server that allows users to efficiently identify the SNPs that will most likely have functional effects. It prioritizes SNPs according to 13 phenotypic risks and putative functional effects, such as changes to the transcriptional level, pre-mRNA splicing, and protein structure. ${ }^{17}$

The functional report on a SNP contains seven sections on the SNP's functional effects, namely:

(i) genomic information, presents the nearby sequence, the alleles and the allele frequency among different ethnic groups;

(ii) functional effects summary, presents the risk assessment;

(iii) transcription regulatory, shows the predicted transcription factor binding sites generated or disrupted by the different SNP alleles;

(iv) alternative splicing regulatory, reports exonic splicing enhancer/silencer motifs changed by the SNP alleles leading to exon skipping or inclusion;

(v) mRNA/protein domain effects, presents all spliced forms of mRNAs and protein variants extracted from GenBank. ${ }^{18}$ The protein domains that the SNP locates in are highlighted;

(vi) protein structure effects, reports whether the SNP may cause a significant structural change in a protein; and

(vii) SwissProt ${ }^{19}$ feature table, provides information regarding other known mutations or variations of the translated protein of mRNAs related to the SNP. ${ }^{17}$

Some of these sections were specific to coding or noncoding SNPs and they will appear or not appear in the functional report accordingly. ${ }^{17}$

\section{Gene information for nsSNPs from Ensembl}

We found the gene related information for the nsSNPs from the Ensembl database. ${ }^{20-22}$ The Ensembl database consists of comprehensive genome information system that features 
Table I SNP rs ID's associated with SMAD I

\begin{tabular}{|c|c|c|c|c|c|c|}
\hline rs43206I28 & rs41837I84 & rs41837|83 & rs41837|82 & rs4l837I8I & rs41837|80 & rs41837179 \\
\hline rs4I837|78 & rs41837I77 & rs41837176 & rs4l837|75 & rs41837I74 & rs41837|73 & rs41837172 \\
\hline rs4l837|7| & rs41837I70 & rs41837169 & rs41837|68 & rs41837I67 & rs41837166 & rs41837I65 \\
\hline rs4I837|64 & rs41837I63 & rs4l837|62 & rs4l837|6I & rs41837I60 & rs41837I59 & rs4I837|58 \\
\hline rs41837|57 & rs4I837I56 & rs41837/55 & rs41837I54 & rs41837I53 & rs41837I52 & rs4l837|5I \\
\hline rs41837|50 & rs4I837I49 & rs4l837|48 & rs41837|47 & rs4I837|46 & rs41837|45 & rs4I837|44 \\
\hline rs4|837|43 & rs41837|42 & rs4|837|4| & rs4|837|40 & rs41837|39 & rs41837|38 & rs41837|37 \\
\hline rs I6383403 & rs16383402 & rsl638340I & rsI6383400 & rs16383399 & rsI6383398 & rs16383397 \\
\hline rs 15529039 & rsI5529035 & rs 15529034 & rsI5529032 & rsI5529030 & rsI5529027 & rsI5529025 \\
\hline rsI5529023 & rsI552902I & rs 15529018 & rsI55290I6 & rs 15529014 & rsI55290II & rsI5529009 \\
\hline rsI5529006 & rsI5529004 & rs 15529002 & rsI5528997 & rsI5528993 & rsl5528990 & rsI5528988 \\
\hline rsI5528985 & rsI5528983 & rs 15528980 & rsI5528978 & rs|5528976 & rsI5528973 & rsI552897| \\
\hline rsI5528968 & rsI5528966 & rs|444587| & rsI4445870 & rs|4445869 & rs|4445868 & rs|4445867 \\
\hline rs I4445866 & rs|4445865 & rs 14445864 & rsI4445863 & rs|4445862 & rs|444586I & rs|4445860 \\
\hline rs 14445859 & rs|4445858 & rs 14445857 & rs|4445856 & rs|4445855 & rs|4445854 & rs|4445853 \\
\hline rs 14445852 & rs|444585। & rs|4445850 & rs|4445849 & rs|4445848 & rs|4445847 & rs|4445846 \\
\hline rs 14445845 & rs|4445844 & rs I4445843 & rsl4445842 & rs|444584I & rs|4445840 & rs|4445839 \\
\hline rs I4445838 & rs|4445837 & rs 14445836 & rsl4445835 & rs|4445834 & rs|4445833 & rs|4445832 \\
\hline rs I444583I & rs|4445830 & rs 14445829 & rsI4445828 & rs|4445827 & rs|4445826 & rs|4445825 \\
\hline rs 14445824 & rs|4445823 & rs I4445822 & rs|444582I & rs|4445820 & rsl44458I9 & rs|44458I8 \\
\hline rsI35I3493 & rs|35|3492 & rs 13513491 & rs 13513490 & rs|35|3489 & $\mathrm{rs}|35| 3488$ & rs|35I3487 \\
\hline rs I35I3486 & rs 10722400 & rs 13455744 & rsl3455743 & rs|3455742 & rs52353258 & rs49393035 \\
\hline rs48744858 & rs40324585 & rs39689443 & rs39378399 & rs38904637 & rs388908I3 & rs3886I705 \\
\hline rs38837210 & rs38736|3| & rs3872886I & rs38673698 & rs38672482 & rs38598229 & rs38594II4 \\
\hline rs38586608 & rs3857|957 & rs38478504 & rs384087I0 & rs38407259 & rs3838II45 & rs38379589 \\
\hline rs38379316 & rs38357614 & rs383432II & rs38319122 & rs38304454 & rs3828677I & rs38216312 \\
\hline rs3819830I & rs38151706 & rs 38122743 & rs38104923 & rs38084479 & rs38069777 & rs38046037 \\
\hline rs38028566 & rs38028025 & rs3800937I & rs37999190 & rs37996620 & rs37970866 & rs37890809 \\
\hline rs37857319 & rs37844585 & rs37829955 & rs37829805 & rs3782024I & rs37798728 & rs37776122 \\
\hline rs37769262 & rs3775I97I & rs3772316I & rs37678|38 & rs37670970 & rs37662I43 & rs3762228I \\
\hline rs37606105 & rs3760I352 & rs37595569 & rs37592912 & rs37570279 & rs37565989 & rs37557932 \\
\hline rs37547978 & rs37529033 & rs $375 \mid 2483$ & rs37502918 & rs37462850 & rs374662363 & rs37447335 \\
\hline rs374247II & rs37395093 & rs37380944 & rs37372985 & rs37358262 & rs37346703 & rs37344855 \\
\hline rs373280I3 & rs37316393 & rs37311870 & rs37278533 & rs37227073 & rs37197406 & rs37I85I97 \\
\hline rs37183904 & rs37176820 & rs37173505 & rs371441214 & rs37138939 & rs37/33086 & rs37095I2I \\
\hline rs37078372 & rs37072603 & rs 37055222 & rs37049288 & rs37041092 & rs37036754 & rs37035385 \\
\hline rs37026804 & rs37021065 & rs 37014646 & rs36984II6 & rs3698I578 & rs36974734 & rs3696|475 \\
\hline rs36935369 & rs36934698 & rs 36926658 & rs36915208 & rs369/3/22 & rs36912217 & rs36910109 \\
\hline rs36904384 & rs36854I39 & rs 36836173 & rs36826819 & rs36807995 & rs36785399 & rs36781909 \\
\hline rs3678I325 & rs36780I56 & rs36773895 & rs36770|38 & rs36763326 & rs36756065 & rs36734|44 \\
\hline rs367204I7 & rs36693953 & rs36682785 & rs36678280 & rs36676848 & rs36652809 & rs36628I23 \\
\hline rs36623863 & rs36620169 & rs36616718 & rs36604460 & rs36604I73 & rs36598919 & rs36592I25 \\
\hline rs36590740 & rs36589398 & rs36589170 & rs36587668 & rs36577742 & rs36574562 & rs36574I54 \\
\hline rs36574562 & rs36574I54 & rs 36550928 & rs36548387 & rs36543793 & rs36550928 & rs36548387 \\
\hline rs36543793 & rs36518106 & rs36516399 & rs36513012 & rs36500074 & rs36499024 & rs36485545 \\
\hline rs3647|433 & rs36463229 & rs36433।I55 & rs364I7697 & rs36409573 & rs36392860 & rs36385065 \\
\hline rs36380669 & rs36378657 & rs 36374178 & rs36372803 & rs36355585 & rs36355433 & rs36355396 \\
\hline rs36343803 & rs36336450 & rs36334006 & rs36308II6 & rs36307375 & rs36304409 & rs3627383I \\
\hline rs36262476 & rs36258235 & rs $1347 \mid 524$ & rs6320822 & rs6320755 & rs6320308 & rs6320203 \\
\hline rs6157027 & rs4II82I87 & rs4l| 62462 & rs $41 \mid 27100$ & rs41097I88 & rs41052054 & rs41042179 \\
\hline rs4100I288 & rs40914529 & rs 40829302 & rs $4080 \mid 804$ & rs 40785650 & rs407/8880 & rs407|4934 \\
\hline rs 40707256 & rs40699558 & rs 40696624 & rs40624834 & rs40600779 & rs2243834I & rs22438340 \\
\hline rs22438338 & rs22435243 & rs 22435242 & rs22430308 & rs22429717 & rs22429/43 & rs22423498 \\
\hline rs22423496 & rs22422179 & rs 22422178 & rs22420I43 & rs22416419 & rs224I3764 & rs22410926 \\
\hline rs 22408159 & rs22407I29 & rs $22407 / 28$ & rs22407/27 & rs22407II26 & rs22407II25 & rs22407/24 \\
\hline rs22407II0 & rs22407109 & rs 22407108 & rs22407I07 & rs22407I06 & rs22407I05 & rs22407I04 \\
\hline rs22407/03 & rs22407I0I & rs22407094 & rs22407093 & rs22407092 & rs2240709। & rs22407090 \\
\hline rs22407089 & rs22407088 & rs 22407087 & rs22407086 & rs22407079 & rs22407078 & rs22407077 \\
\hline
\end{tabular}


Table I (Continued)

\begin{tabular}{|c|c|c|c|c|c|c|}
\hline rs22407076 & rs22407075 & rs22407074 & rs22407073 & rs22407072 & rs2240707I & rs22407070 \\
\hline rs22407064 & rs22407062 & rs22407060 & rs22407059 & rs22407057 & rs22407056 & rs22407055 \\
\hline rs22407054 & rs22407052 & rs22407049 & rs22407047 & rs22407045 & rs22407044 & rs22407043 \\
\hline rs22407042 & rs2240704I & rs2240506I & rs22403II5 & rs2240I316 & rs22399442 & rs22398238 \\
\hline rs223982 II & rs22398210 & rs22379962 & rs22379948 & rs22379160 & rs22364266 & rs223642I 2 \\
\hline rs2236368I & rs2235353I & rs22353529 & rs22349453 & rs22349452 & rs2234945I & rs22348997 \\
\hline rs22348996 & rs9197660 & rs9197659 & rs9190264 & rs9190263 & rs9159692 & rs915969I \\
\hline rs9159690 & rs9159689 & rs9159688 & rs912790I & rs9l 27900 & rs9l I3580 & rs9082059 \\
\hline rs9082058 & rs9015277 & rs9015276 & rs9015275 & rs896764I & rs8967640 & rs8958253 \\
\hline rs8958252 & rs894823 I & rs8948230 & rs8948229 & rs8948228 & rs89| 6864 & rs89047I5 \\
\hline rs88786I9 & rs88786I 8 & rs88786I7 & rs88786I6 & rs88786I5 & rs887||49 & rs887||48 \\
\hline rs887| |47 & rs882|428 & rs86I 6390 & rs86I 6389 & rs8548I 36 & rs8548I 35 & rs8548I 34 \\
\hline rs8548I 33 & rs8434954 & rs8434953 & rs8434952 & rs843495 I & rs84I 2032 & rs26109380 \\
\hline rs25383970 & rs25350003 & rs25327873 & rs25326101 & rs2532I520 & rs25306350 & rs25297286 \\
\hline rs25294I2I & rs25292078 & rs25285454 & rs25281219 & rs2527906I & rs25263I7I & rs2524I6I4 \\
\hline rs25239333 & rs25238019 & rs25231997 & rs24891022 & rs62446864 & rs62446863 & rs62446862 \\
\hline rs61613428 & rs6II 28823 & rs61098313 & rs60956809 & rs60584748 & rs60532382 & rs60323633 \\
\hline rs60103805 & rs59784627 & rs59525016 & rs59034773 & rs5889|283 & rs585727I3 & rs5844I52I \\
\hline rs57305857 & rs572321I4 & rs56022562 & rs558II804 & rs5573I773 & rs4I27599I & rs4I 275988 \\
\hline rs36125I63 & rs35821025 & rs357II502 & rs35482863 & rs35425I65 & rs35302357 & rs35241091 \\
\hline rs35I5I755 & rs35045679 & rs34957085 & rs3488566I & rs34725384 & rs34668775 & rs34495080 \\
\hline rs34457367 & rs3423082I & rs34I49226 & rs28937323 & rs28937322 & rs28936972 & rs17159296 \\
\hline rsl7|5929I & rsI7I59289 & rsI7I59287 & rsI7I59280 & rsI7I59279 & rsl7159275 & rs I7I59273 \\
\hline rsI7I59272 & rsI7I59267 & rsI7I59262 & rs60939160 & rs I 6875290 & rsI7I59297 & rs I3228758 \\
\hline rs| 3228097 & rs|3228094 & rs|3228006 & rs|32267/8 & rsI2532057 & rsl| 982890 & rsII98II06 \\
\hline rs I I980069 & rsII 979352 & rsI I 976576 & rs56650l46 & rsII76I080 & rsII553503 & rsII553502 \\
\hline rsII553498 & rsII553497 & rsI|465024 & rs35809100 & rs I I 454228 & rs 17778009 & rs60345I84 \\
\hline rsl095I27I & rsl0487700 & rsl0487699 & rsl028049| & rs6I 212867 & rsl0265589 & rs I0264469 \\
\hline rs I0249885 & rsl0239660 & rs57200522 & rsI0239537 & rs10233132 & rsl0229836 & rs7808770 \\
\hline rs6970807 & rs69500I5 & rsl0347965 & rs6947982 & rs6945586 & rs6945546 & rs6945475 \\
\hline rs6945223 & rs6945035 & rs64622I4 & rs40002I0 & rs388664l & rs3840589 & rs|l403655 \\
\hline rs3840588 & rs3807633 & rsl0386I94 & rs9792086 & rs56638865 & rs3807632 & rs2970504 \\
\hline rs2952805 & rs2952804 & rs2952795 & rs446I 798 & rs2893390 & rs2893389 & rs2893388 \\
\hline rsl03780|4 & rs373543I & rs2709809 & rs2709806 & rs380763I & rsI7700042 & rs57637303 \\
\hline rs2709778 & rs6076I314 & rs2709772 & rs270977I & rs2529442 & rs60428326 & rs252944I \\
\hline rs3779252 & rs2529439 & rsl I55350I & rs2529438 & rs3764826 & rs57476542 & rs2527880 \\
\hline rs60677610 & rs2527879 & rs2527878 & rs 10378720 & rs2270025 & rs224050I & rs58255920 \\
\hline rsl0378822 & rs224040I & rs60101002 & rs2240400 & rs34886|42 & rs2230310 & rs22|4837 \\
\hline rs219024I & rs2074779 & rs2072236 & rs I986757 & rs56754355 & rsI03380I 7 & rs I986756 \\
\hline rs5947/849 & rsI558064 & rs|468402 & rs I74I3053 & rs3189753 & rsII30653 & rs3189564 \\
\hline rsII553500 & rsI7856227 & rsI 7856223 & rs I049402 & rs3189560 & rs I049399 & rs2709804 \\
\hline rs758000 & rs61005429 & rs728539 & rs722423 & rs17159306 & rsII30593 & rs3189633 \\
\hline rs22303 II & rs2070205 & rs I80I345 & rs I049584 & rs3 I 89770 & rs4593 & rs62343509 \\
\hline rs62343505 & rs62343494 & rs62343493 & rs62343492 & rs6I303468 & rs6III02345 & rs60788260 \\
\hline rs60537353 & rs60369536 & rs60I39040 & rs60073922 & rs60072759 & rs600I4433 & rs59984540 \\
\hline rs59971057 & rs59933736 & rs59909582 & rs59846899 & rs59268619 & rs59193620 & rs59063315 \\
\hline rs59057604 & rs59054276 & rs59029464 & rs58968834 & rs589|3698 & rs58688997 & rs58688046 \\
\hline rs58468364 & rs58339l65 & rs57963489 & rs57797623 & rs57612157 & rs5758I 646 & rs57545372 \\
\hline rs57257834 & rs57044560 & rs56986943 & rs56985736 & rs56837794 & rs56756555 & rs56695028 \\
\hline rs60478333 & rs56250I 24 & rs55948666 & rs55835219 & rs55700163 & rs3600795I & rs35999258 \\
\hline rs35986I6I & rs359566I8 & rs35923324 & rs35882080 & rs35842798 & rs358I37|4 & rs358I 2565 \\
\hline rs35774186 & rs35756866 & rs35747692 & rs35710925 & rs35668194 & rs356I2203 & rs35584992 \\
\hline rs35528395 & rs35483247 & rs35476542 & rs35386763 & rs35281972 & rs3524I 245 & rs35 I 84283 \\
\hline rs35058397 & rs35046379 & rs62343508 & rs34978105 & rs34968029 & rs34959603 & rs34880052 \\
\hline rs34832344 & rs3479|274 & rs34791030 & rs34629544 & rs34610916 & rs34504494 & rs34443486 \\
\hline rs34405 I I6 & rs34373983 & rs34333288 & rs343।8288 & rs34303II6 & rs34I696I5 & rs34I4265I \\
\hline rs56664494 & rs34107624 & rs34082880 & rs34079792 & rs340774I7 & rs340I3277 & rs340II978 \\
\hline
\end{tabular}


Table I (Continued)

\begin{tabular}{|c|c|c|c|c|c|c|}
\hline rs600I50I4 & rs33928689 & rs5795।II97 & rs28607764 & rs 28579035 & rs2854I642 & rs56683612 \\
\hline rs28534855 & rs 28522520 & rs28495533 & rs28464746 & rs 28408896 & rs28397904 & rs I7797966 \\
\hline rs|7797805 & rs 17741593 & rs 17426247 & rs59063334 & rs I702032I & rs 17020317 & rsI7020315 \\
\hline rs|7020313 & rs 17020310 & rsI7020304 & rsI7020302 & rs I7020297 & rsI7020284 & rsI702028I \\
\hline rsI7020269 & rs I7020255 & rs 17020253 & rsI702025I & rs59444418 & rsI7020248 & rsI7020237 \\
\hline rsI7020236 & rs58221759 & rsI7020235 & rsI7020202 & rs59633267 & rsl702020I & rs56640II8 \\
\hline rs58653480 & rs I7020200 & rsl6998662 & rsl6998659 & rs I3435697 & rs|3|49786 & rs|3|4977| \\
\hline rs|3|49754 & rs|3|45825 & rsl3|44I5I & rs|3|38805 & rs58040II3 & rs|3|37598 & rs|3|20843 \\
\hline rs|3||8865 & rs|3113936 & rs13110369 & rs|3|04775 & rs I7426240 & rs 12646702 & rs 12504239 \\
\hline rs589/0572 & rs I 1946962 & rsII946830 & rsII945076 & rs58I66623 & rsII944685 & rsII 944363 \\
\hline rs|l939520 & rsII939179 & rsII938489 & rsII937875 & rsII 937328 & rsII936636 & rsII935522 \\
\hline rs577356|3 & rsII935237 & rs|3|2937| & rsII934722 & rs|l93|456 & rs|3107924 & rsII930852 \\
\hline rs13104519 & rsII 930324 & rsII736932 & rsII725I0I & rs56452565 & rs58073224 & rsII7248I3 \\
\hline rsII547|80 & rsII547|78 & rsII537832 & rs58395I99 & rsII392205 & rsII355954 & rsII302430 \\
\hline rs33926573 & rs II 293293 & rs33995597 & rsII 285972 & rsIII00885 & rsIII00884 & rs17798I52 \\
\hline rs60525943 & rs56482779 & rsIII00883 & rs56920353 & rsIII00882 & rsI07I5854 & rs35752574 \\
\hline rs107|3518 & rsI0694III & rs 10677232 & rs36210455 & rs 10599198 & rs 10557196 & rs33996344 \\
\hline rs10548I53 & rs 10519733 & rsI0033759 & rs9992345 & rs9968492 & rs9685596 & rs9683725 \\
\hline rs769993। & rs7699352 & rs59616816 & rs7698944 & rs7695203 & rs613|4879 & rs7694604 \\
\hline rs7694086 & rs56553319 & rs7689005 & rs7686994 & rs60923728 & rs7685592 & rs7685387 \\
\hline rs7684336 & rs7683590 & rs58089363 & rs768240I & rs768I7I3 & rs7677879 & rs7677863 \\
\hline rs7677I60 & rs76758II & rs59990034 & rs767382I & rs7672606 & rs59927।96 & rs7672306 \\
\hline rs60I92542 & rs7670486 & rs7670403 & rsI7798495 & rs7662543 & rs5666/2II & rs766254I \\
\hline rs5655433I & rs766II62 & rs7658436 & rs7658004 & rs57170185 & rs7655568 & rs74353।I \\
\hline rs|7435952 & rs6854034 & rs61107II7 & rs6852200 & rs61409198 & rs6848098 & rs6845829 \\
\hline rs576I1059 & rs6833260 & rs6829932 & rs682909l & rs68235I3 & rs6822700 & rs68I6306 \\
\hline rs68|3833 & rs6537359 & $\mathrm{rs}|3| 2 \mid 278$ & rsI7797649 & rs6537356 & rs 17426233 & rs57727279 \\
\hline rs6537355 & rs60611965 & rs35037580 & rs5862739 & rs5862738 & rs4395527 & rs3948257 \\
\hline rs3943707 & rs3816967 & rs3775325 & rs3775323 & rs3775322 & rs60III2569 & rs375602I \\
\hline rs57309032 & rs 17741322 & rs2289737 & rsI7227|89 & rs2118438 & rs57644485 & rs206899I \\
\hline rs6II03877 & rs2043779 & rs57261547 & rs20I3367 & rs59728727 & rs $1774 \mid 821$ & rs1992165 \\
\hline rs 1804647 & rs|497|26 & rs|437827 & rs|437826 & rs|37057| & rs5762802I & rsI055440 \\
\hline rs3816966 & rs58I5228I & rsI0I6792 & rs60988923 & rsl01679I & rs6058|465 & rs95964l \\
\hline rs3775324 & rs92026I & rs58547099 & rs768I39 & rs763560 & rs 17798104 & rs7|4|95 \\
\hline rsI5392 & rsl|4l|6. & rsl4270 & rs62343506 & & & \\
\hline
\end{tabular}

an integrated set of genome annotation, databases and other information for chordate and selected model organism and disease vector genomes. ${ }^{20-22}$ The Ensembl genome browser provides visualization for genome annotations, alignments, variation and functional genomics data and supporting additional data integration.

Ensembl gene sets are created using an automated analysis pipeline that has been significantly optimized based on the completeness of the genome sequence. ${ }^{20-22}$

\section{Functional significance of nsSNPs by UTR scan}

The untranslated regions (UTR) functional significance of each SNP was determined using UTResource, an internet based UTR analyzing tool, consisting of a resource of sequence analysis of 5' and 3' UTR of eukaryotic mRNAs
(http://www.ba.itb.cnr.it/BIG/UTRScan). Briefly, two or three sequences of each UTR SNP which have different nucleotide at SNP position were analyzed by UTRscan which looks for UTR functional elements by searching through user submitted sequence data for the patterns defined in the UTRsite collection.

The collection of functional sequence patterns located in 5' or 3' UTR sequences were stored in UTRsite. If different sequences of each UTR SNP were found to have different functional pattern(s), that UTR SNP is predicted to have functional significance. ${ }^{13}$

\section{Modeling wild and mutant protein structures}

We utilized the SWISS-MODEL ${ }^{23-25}$ workspace, a web-based integrated service dedicated to protein structure homology 
modeling for modeling the native and mutant protein structures. Swiss-Model assists and guides the user in building protein homology models at different levels of complexity. A personal working environment is provided for each user where several modeling projects can be carried out in parallel.

The program builds a homology model based on four main steps:

(1) identification of structural template(s),

(2) alignment of target sequence and template structure(s),

(3) model building and

(4) model quality evaluation.

\section{3-D viewing and RMSD calculations}

The Swiss PDB viewer (Deep View) version 4.01 was used for viewing the modeled structures and for calculation of the root mean square deviation (RMSD) between the native and mutant structures. ${ }^{26}$ DeepView is an application that provides a user friendly interface to analyze several proteins at the same time. The proteins can be superimposed in order to deduce structural alignments and compare their active sites or any other relevant parts. Amino acid mutations, hydrogen bonds, angles and distances between atoms can be obtained using Deep View. ${ }^{26}$

\section{Results and Discussion}

We used the SIFT tool to predict whether an amino acid substitution at a particular position in a protein molecule will have a phenotypic effect. We find that of 1045 rs IDs associated with SMAD1 gene, only two, rs1804647 and rs17159287, are non-synonymous.

SIFT gives the results of these nsSNPs with their amino acid change and the tolerance index score which predicts the functional impact of the protein of these SNPs. The lesser the score, the more functional impact, ie, the corresponding SNP is predicted to be damaging. Of these two SNPs, rs 1804647 is predicted to be damaging with a tolerance index score of 0.01 . (Score of $\leq 0.05$ is the cut-off to identify damaging SNPs $)^{11-13}$ as shown in Table 2. The nucleotide change is in the 510 position where AAA is replaced with GAA ie,
Adenine to Guanine resulting in the substitution of amino acid Lysine to Glutamate. (The base represented in bold caption is the nsSNP).

We employed the PolyPhen based methodology ${ }^{14-16}$ to compute the position-specific independent counts (PSIC) score difference of the two identified nsSNPs (rs1804647 and rs17159287) to assess the functional impact of the amino acid substitution predicted by SIFT. To evaluate the ability of the PolyPhen method to distinguish between damaging and neutral amino acid types; we applied it to the data on known deleterious mutations and to the data on species divergence. ${ }^{15}$ The deleterious data set consists of natural replacements known to cause disease phenotypes, variants observed in individuals affected by genetic disorders and artificial replacements known to damage structure, function and the stability of a protein. ${ }^{15}$ Score of $\geq 1.5$ is the cut-off to identify damaging SNPs by Polyphen. SNP with PSIC score difference of higher value is predicted to be damaging. We found that of the two nsSNPs, rs1804647 is predicted to be damaging with a PSIC score difference of 1.852, as depicted in Table 3.

FASTSNP was employed to efficiently identify and prioritize high risk nsSNPs according to their phenotypic risks and putative functional effects. ${ }^{17}$ FASTSNP rates the phenotypic deleterious risks from 0 to 5 point scale; 0 representing no known effect and 5 indicating very high damaging effect, while a score of 3-4 indicates moderate to high damaging effect. ${ }^{17}$ We found rs 17159287 to have low to medium level of risk, whereas rs 1804647 was identified to have significantly damaging effect with a score in the range of 3-4; as shown in Table 4. This may be due to splicing regulation functional effect by possibly breaking the exonic splicing enhancer/silencer binding site in the coding sequence leading to abolished protein domain. ${ }^{17}$

Gene related information the non-synonymous SNPs associated with SMAD1, with their corresponding change in alleles and amino acids were obtained from Ensembl database and we found three of the 1045 associated with SAMD1 listed as nsSNPs. These are: rs1804647, rs61748163; and rs62343507, depicted in Table 5.

Table 2 nsSNPs predicted by SIFT

\begin{tabular}{lllllll}
\hline SNP ID & $\begin{array}{l}\text { Amino } \\
\text { acid change }\end{array}$ & Protein & $\begin{array}{l}\text { Damaging } \\
\text { amino acid }^{\mathbf{a}}\end{array}$ & Damage & $\begin{array}{l}\text { Score } \\
\text { sequence IC }\end{array}$ \\
\hline rs1804647 & E32K & NP_00589I & E & YES & 0.01 & 3.29 \\
rs17I59287 & Q334R & NP_002038 & Q & NO & 0.59 & 2.44 \\
\hline
\end{tabular}

aAbbreviations: E, Glutamate; K, Lysine; Q, Glutamine; R, Arginine. 
Table 3 nsSNPs predicted by PolyPhen

\begin{tabular}{lllllll}
\hline SNP ID & Protein & Position & $\begin{array}{l}\text { Amino } \\
\text { acid I }\end{array}$ & $\begin{array}{l}\text { Amino } \\
\text { acid 2 }\end{array}$ & PSICa & Prediction \\
\hline rsI7I59287 & P4I250 NP_002038.I & 388 & R & Q & 0.298 & Benign \\
rsI804647 & I. Q15797 NP_00I003688.I & 32 & K & E & 1.852 & Possibly damaging \\
& 2. Q15797 NP_00589I.I & 32 & $\mathrm{~K}$ & $\mathrm{E}$ & 1.852 & Possibly damaging \\
\hline
\end{tabular}

aPosition Specific Independent Count.

There is a significant correlation between all the SNP tools with respect to rs1804647 and hence this SNP might be expected to have high deleterious impact on the function of the bone morphogenesis process. The other two SNPs (rs61748163 and rs62343507) did not show any result with other tools used and hence, we did not consider them for our study.

To determine the functional significance of the nsSNP rs 1804647 , we used UTRScan and found a change in a pattern in 3' UTR region. Polymorphism in the 3' UTR region may affect gene expression by affecting RNA half-life or influencing ribosomal translation of mRNA. ${ }^{27}$

The functional element change related to this nsSNP was found to be 15-lipoxygenase differentiation control element (15-LOX-DICE). 15-LOX-DICE controls 15-LOX synthesis which catalyses the degradation of lipids and is an important factor responsible for the degradation of mitochondria during reticulocyte maturation. ${ }^{13}$ The mutation Lysine $\rightarrow$ Glutamate is observed in the protein due to nucleotide change in the coding sequence of the SNP in 510 position where AAA is replaced with GAA. From this we infer that the nsSNP, rs1804647 is significantly damaging which might result in osteoporosis.

The nsSNP, rs 17159287 has tolerance index score of 0.59 with SIFT, a PSIC score difference of 0.298 with PolyPhen, a relatively low risk score of 2-3 with FASTSNP and was not listed in Ensembl database. Hence we did not consider this nsSNP to be significantly damaging.

SNP rs61748163 has amino acid change from Serine to Asparagine, both are of same properties and showed

Table 4 nsSNPs predicted by FAST SNP

\begin{tabular}{llll}
\hline SNP ID & $\begin{array}{l}\text { Position of } \\
\text { change in } \\
\text { amino acid }\end{array}$ & Level of risk & $\begin{array}{l}\text { Possible } \\
\text { functional effect }\end{array}$ \\
\hline rsI804647 & 32 & $\begin{array}{l}3-4 \\
\text { (moderate to high) }\end{array}$ & Splicing regulation \\
rsI7I59287 & 388 & $\begin{array}{l}2-3 \\
\text { (low to medium) }\end{array}$ & Splicing regulation \\
\hline
\end{tabular}

no change in structure when native and mutant proteins were modeled. SNP, rs62343507 has amino acid change from Tryptophan to Arginine, also showed no change in structure when native and mutant proteins were modeled. Hence, we did not consider these nsSNPs (rs61748163 and rs62343507) listed in Ensembl to be significantly damaging and they did not show any results with other bioinformatics tools.

To assess the 3-D structural change brought about by the nsSNP rs1804647, we performed structural analysis comparing the native and mutant protein structures. The native structure for protein Q15797 and mutant structure for nsSNP rs 1804647 were modeled using SWISS-MODEL. To analyze their structural differences, superimposition of both the structures was done using the SWISS PDB Viewer tool and we found an RMSD of $2.95 \AA$ between the native and mutant protein structures as shown in Figure1. This nsSNP also showed a high PSIC score difference of 1.852. Hence, there might be a considerable change in the structure because of this nsSNP. Even though, there is only one amino acid change, the change in amino acid is of the different type. Here the amino acid Lysine is a cationic residue in the native protein and the substituted residue in the mutant protein molecule is Glutamate which is an anionic residue. Due to the complete change in charges brought about by this mutation, there seems to be a considerable change in structure.

The function of a protein is dependent on its 3-D structure and shape which is determined by its primary structure or sequence of amino acids. Since there is a change in amino acid sequence due to the single nucleotide polymorphism,

Table 5 nsSNPs predicted by ENSEMBL database

\begin{tabular}{lllll}
\hline AA position & SNP ID & SNP type & Alleles $^{\mathrm{a}}$ & $\begin{array}{l}\text { Amino } \\
\text { acid } \\
\text { change }^{\mathrm{a}}\end{array}$ \\
\hline 32 & $\mathrm{rs} / 804647$ & Non-synonymous & A/G & $\mathrm{K}, \mathrm{E}$ \\
281 & $\mathrm{rs} 61748163$ & Non-synonymous & A $/ \mathrm{G}$ & $\mathrm{S}, \mathrm{N}$ \\
358 & $\mathrm{rs} 62343507$ & Non-synonymous & $\mathrm{C} / \mathrm{T}$ & $\mathrm{W}, \mathrm{R}$ \\
\hline
\end{tabular}

aAbbreviations: A, Adenine; G, Guanine; C, Cytosine; T, Thymidine; K, Lysine; E, Glutamate; S, Serine; N, Asparagine; W, Tryptophan; R, Arginine. 


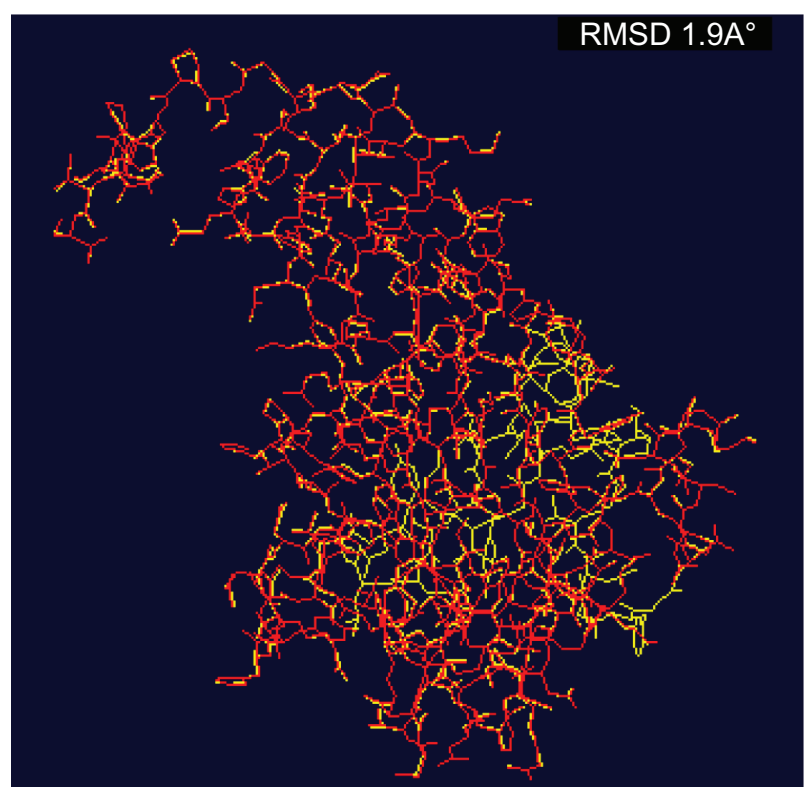

Figure I Superimposed structures of native (yellow) and mutant (red) proteins.

non functional protein may be produced which will lead to altered overall function and hence this particular nsSNP may be responsible for a defective bone morphogenic protein and result in osteoporosis.

Results obtained from this in-silico study open new prospects for understanding osteoporosis and its consequences. These results, if correlated with clinical data, will be very useful in understanding the genetics of osteoporosis.

The methodology used to examine the effects of SNPs using a succession of tools could be structured and proposed as a standard screening process for SNPs' effects assessment on genes.

\section{Conclusion}

The nsSNP rs 1804647 is predicted to be damaging by SIFT, PolyPhen, FASTSNP and listed as deleterious in Ensembl database. The change of nucleotide in 510th position from A to $\mathrm{G}(\mathbf{A A A} \rightarrow \mathbf{G A A})$ results in the amino acid change from Lysine to Glutamate. Since the change of amino acid residues is of opposite charge it may lead to conformational change of the three dimensional structure of the protein and hence result in altered function.

This observation can be considered as a good beginning for further investigations to study the genetic mechanisms behind osteoporosis, and when considered along with different clinical patterns of osteoporosis will result in more definitive evidence for the genetic origin of osteoporosis.

\section{Disclosure}

The authors report no conflicts of interest in this work.

\section{References}

1. Cline RR, Farley JF, Hansen RA, et al. Osteoporosis beliefs and antiresorptive medication use. Maturitas. 2005;50(3):196-208.

2. Baylink DJ, Strong DD, Mohan S. The diagnosis and treatment of osteoporosis: future prospects. Molecular Medicine Today. 1999;5(3):133-140.

3. Reginster JY, Burlet N. Osteoporosis: A still increasing prevalence. Bone. 2006;38(2 Suppl 1): S4-S9.

4. Ferrari S. Human genetics of osteoporosis. Best Practice and Research Clinical Endocrinology \& Metabolism. 2008;22(5):723-735.

5. Liu YJ, Shen H, Xiao P, et al. Molecular genetic studies of gene identification for osteoporosis: a 2004 update. Journal of Bone and Mineral Research. 2006;21(10):1511-1535.

6. Bajdik CD, Kuo B, Rusaw S, et al. CGMIM: automated text-mining of Online Mendelian Inheritance in Man (OMIM) to identify genetically-associated cancers and candidate genes. BMC Bioinformatics. 2005;78(6): 1-7.

7. Gajendran VK, Lin JR, Fyhrie DP. An application of bioinformatics and text mining to the discovery of novel genes related to bone biology. Bone. 2007;40(5):1378-1388

8. Mah JTL, Chia KS. A gentle introduction to SNP analysis: resources and tools. J Bioinfo Comp Biol. 2007;5(5):1123-1138.

9. Ross S, Hill CS. How the Smads regulate transcription. The Int $J$ of Biochem. Cell Biol. 2008;40(3):383-408.

10. Sherry ST, Ward MH, Kholodov M, et al. dbSNP: The NCBI database of genetic variation. Nucleic Acids Res. 2001;29(1): 308-311.

11. Ng CP, Henikoff S. SIFT: Predicting amino acid changes that affect protein function. Nucleic Acids Res. 2003;31(13):3812-3814.

12. Ng CP, Henikoff S. Predicting deleterious amino acid substitutions. Genome Res. 2001;11:863-874.

13. Shen J, Deininger PL, Zhao H. Applications of computational algorithm tools to identify functional SNPs in cytokine genes. Cytokine. 2006;35(1-2):62-66.

14. Ramensky V, Bork P, Sunyaev S. Human non-synonymous SNPs: Server and survey. Nucleic Acids Res. 2002;30(17):3894-3900.

15. Sunyaev S, Ramensky V, Koch I, et al. Prediction of deleterious human alleles. Hum Mol Genet. 2001;10(6):591-597.

16. Sunyaev S, Ramensky V, Bork P. Towards a structural basis of human non-synonymous single nucleotide polymorphisms. Trends Genet. 2000;16(5):198-200.

17. Yuan HY, Chiou JJ, Tseng WH, et al. FASTSNP: an always up-to-date and extendable service for SNP function analysis and prioritization. Nucleic Acids Res. 2006;34(Web Server):W635-W641.

18. Benson DA, Karsch-Mizrachi I, Lipman DJ, et al. GenBank: update. Nucleic Acids Res. 2004;32(Database):D23-D26.

19. Boeckmann B, Bairoch A, Apweiler R, et al. The Swiss-Prot protein knowledgebase and its supplement TrEMBL in 2003. Nucleic Acids Res. 2003;31(1):365-370.

20. Hubbard TJP, Aken BL, Ayling S, et al. Ensembl 2009. Nucleic Acids Res. 2009;37(Database):D690-D697.

21. Flicek P, Aken BL, Beal K, et al. Ensembl 2008. Nucleic Acids Res. 2008;36(Database):D707-D714.

22. Hubbard T, Barker D, Birney E, et al. The Ensembl genome database project. Nucleic Acids Research. 2002;30(1):38-41.

23. Arnold K, Bordoli L, Kopp J, et al. The SWISS-MODEL Workspace: A web-based environment for protein structure homology modelling. Bioinformatics. 2006;22(2):195-201.

24. Kopp J, Schwede T. The SWISS-MODEL Repository of annotated threedimensional protein structure homology models. Nucleic Acids Res. 2004;32(Database):D230-D234. 
25. Schwede T, Kopp J, Guex N, et al. SWISS-MODEL: an automated protein homology-modeling server. Nucleic Acids Res. 2003;31(13):3381-3385

26. Guex N, Peitsch MC. SWISS-MODEL and the Swiss-PdbViewer: An environment for comparative protein modeling. Electrophoresis. 1997;18(15):2714-2723.
27. van Deventer SJH. Cytokine and cytokine receptor polymorphisms in infectious disease. Intensive Care Med. 2000;26(13):S98-S102.

Open Access Bioinformatics

\section{Publish your work in this journal}

Open Access Bioinformatics is an international, peer-reviewed, open access journal publishing original research, reports, reviews and commentaries on all areas of bioinformatics. The manuscript management system is completely online and includes a very quick and fair
Dovepress

peer-review system. Visit http://www.dovepress.com/testimonials.php to read real quotes from published authors. 